\title{
The Incidence and Prevalence of Thyroid Disease Do Not Differ in the Multiple Sclerosis and General Populations: A Validation Study Using Administrative Data
}

\author{
Ruth Ann Marrie ${ }^{a}$ b Bo Nancy Yu ${ }^{b}$ Stella Leung ${ }^{b}$ Lawrence Elliott ${ }^{b}$ \\ Sharon Warren ${ }^{c}$ Christina Wolfson ${ }^{d, e}$ Helen Tremlett $^{f}$ John Fisk $^{g}$ \\ James Blanchard ${ }^{b}$ \\ Departments of a Internal Medicine and ${ }^{\mathrm{b} C}$ Community Health Sciences, University of Manitoba, Winnipeg, Man., \\ 'Faculty of Rehabilitation Medicine, University of Alberta, Edmonton, Alta., ${ }^{\mathrm{d}}$ Department of Epidemiology and \\ Biostatistics, McGill University, and eResearch Institute of the McGill University Health Centre, Montreal, Que., \\ fDepartment of Medicine (Neurology), University of British Columbia, Vancouver, B.C., and ${ }^{9}$ Departments of \\ Psychiatry and Medicine, Dalhousie University, Halifax, N.S., Canada
}

\section{Key Words}

Multiple sclerosis - Autoimmune thyroid disease •

Administrative data $\cdot$ Validation $\cdot$ Prevalence

\begin{abstract}
Background: Prior studies of a possible increased risk of autoimmune thyroid disease (AIT) in multiple sclerosis (MS) are inconsistent. We aimed to validate and apply administrative case definitions for the surveillance of AIT in MS. Methods: We used administrative health data to identify 4,192 persons with MS and an age-, sex- and geographically matched general population cohort $(n=20,940)$. We developed case definitions for AIT using International Classification of Disease- $9 / 10$ codes and prescription claims, compared them to medical records and applied them to estimate the incidence and prevalence of AIT. Results: When compared to medical records, the administrative case definition using $\geq 1$ hospital or $\geq 2$ physician or $\geq 2$ prescription claims had a sensitivity of $73.5 \%$ and specificity of $98.4 \%$. In 2005 , the age-adjusted prevalence of AIT was $9.51 \%$ [95\% confidence interval (Cl) $8.46-10.6]$ in the MS population and $8.56 \%(95 \% \mathrm{Cl} 8.11-9.02)$
\end{abstract}

in the general population. The age-adjusted incidence of AIT per 100,000 persons per year was 422.8 (95\% Cl 204.4-641.3) in the MS population and $407.7(95 \% \mathrm{Cl} 308.5-506.9)$ in the general population. From 1996 to 2005, the prevalence of AIT rose in both populations. Conclusion: Administrative data can be used for surveillance of AIT in MS. The incidence and prevalence of thyroid disease are similar in the MS and general populations.

Copyright $\odot 2012$ S. Karger AG, Basel

\section{Introduction}

Several studies have evaluated the occurrence of autoimmune thyroid disease (AIT) in the multiple sclerosis (MS) population, reporting prevalences ranging from 0.92 to $13.9 \%[1,2]$. Some of these studies suggested that

The results and conclusions presented are those of the authors. No official endorsement by Manitoba Health is intended or should be inferred.

\section{KARGER \\ Fax +4161306 1234 \\ E-Mail karger@karger.ch}

www.karger.com
(C) $2012 \mathrm{~S}$. Karger AG, Basel

$0251-5350 / 12 / 0392-0135 \$ 38.00 / 0$

Accessible online at:

www.karger.com/ned
Ruth Ann Marrie, MD, PhD

Health Sciences Center, GF-533

820 Sherbrook Street

Winnipeg, MB R3A 1R9 (Canada)

Tel. +1 204787 4951, E-Mail rmarrie@hsc.mb.ca 
AIT is more common in individuals with MS as compared to the findings expected for the general population [3-5]; however, most of these studies did not use contemporaneous control groups. Further, few studies were population-based and even these have produced inconsistent findings [4-6].

The development of methods for surveillance of AIT is an increasingly important issue in MS given the potential adverse effects of disease-modifying therapy. Interferon-beta may be associated with an increased risk of thyroid dysfunction and autoimmunity [7]. Alemtuzumab is associated with an increased risk of Graves' disease of up to $23 \%$ [8]. Given that the adverse effects of medication may be delayed following exposure [9] and are underreported [10], better surveillance methods are needed. Administrative (health claims) data may support population-based disease surveillance and pharmacovigilance but must be validated for research purposes [11].

We aimed to develop and validate administrative case definitions for the surveillance of AIT in MS which would support future pharmacovigilance studies. We applied these definitions to estimate the incidence and prevalence of AIT in the MS population as compared to the general population over a 20 -year period.

\section{Methods}

We conducted a population-based analysis of administrative (health claims) data for the period 1984-2005 using a matched cohort design.

\section{Administrative Data}

The Canadian province of Manitoba has a population of approximately 1.2 million. Manitoba Health $(\mathrm{MH})$, a provincial government department, provides health care services to $98 \%$ of the population [12]. Since 1984, all provincial residents have been assigned a unique personal health identification number (PHIN) which is attached to all hospital, physician and prescription claims submitted to MH. In addition to the PHIN, each physician claim includes the date of service and the three-digit International Classification of Disease (ICD)-9-Clinical Modification code for one (most responsible) physician-assigned diagnosis. Each hospital discharge abstract (claim) includes the admission date, discharge date and up to 16 discharge diagnoses using ICD-10-Canadian Enhancement codes. Prior to 2004, discharge diagnoses were recorded using five-digit ICD-9-Clinical Modification codes. Since 1996, the Drug Programs Information Network has captured all outpatient prescription drug dispensations for Manitoba residents including the date, drug name and drug identification number.

\section{Study Populations}

As detailed elsewhere, we developed and validated an administrative case definition for MS [13]. Using this definition, we identified all persons with MS in Manitoba as follows: from 1984 to 1997 , cases were persons with $\geq 7$ hospital or physician claims for MS, and from 1998 to 2007, cases were persons with $\geq 3$ hospital, physician or prescription claims for MS. After excluding persons with any ICD-9/10 diagnostic code for any demyelinating disease (i.e. 377.3, 323.82, 323, 341.9, 341.0, H46, G37, G35, G36), we concurrently identified a non-MS (control) cohort from the general population, individually matched on sex, year of birth and region of residence (postal code) to the MS cohort, with up to 5 controls for each case [13]. For each person with MS, we assigned a date of diagnosis based on the date of the first health claim for demyelinating disease, and the same (index) date was assigned to the matched controls.

\section{Validation Cohort}

In our prior study which validated the administrative definition of MS [13], 594 participants with and without MS consented to linkage of their administrative and medical records data. In a subsequent study which validated a self-report questionnaire for comorbidity, 227 participants followed in the only MS clinic in Manitoba agreed to linkage of their administrative and clinical data [14]. In both studies, trained abstractors, blinded to the administrative data findings, used the same standardized data collection form to abstract comorbidity data from the medical records following training by the senior investigator (R.A.M.). The senior investigator conducted random audits of the charts being reviewed to ensure ongoing consistency of data collection. Together, these studies provided a cohort of 430 unique persons with definite MS [15] for whom administrative case definitions could be compared to medical records. A sample size of 400 is adequate to detect a $\kappa$ of $\geq 0.60$ for a condition with at least $10 \%$ prevalence where the null hypothesis is that $\kappa=0.40, \alpha=0.05$ and $\beta=0.20$.

\section{Development and Validation of Administrative Case}

Definitions

For this study, we focused on AIT because most studies have suggested that it affects more than $5 \%$ of persons with MS, making it potentially important at the population level. Also, it may be an adverse effect of some MS treatments such that a validated algorithm along with population estimates of incidence and prevalence will aid future pharmacovigilance studies. Malignancies may also be of interest as an adverse effect of emerging therapies, but thyroid cancer has not been a concern to date and is quite rare in the general population [16]. Thus, the small size of our validation cohort precluded evaluation of this condition. We selected ICD-9/10 codes for autoimmune hyperthyroidism $(242,376.21$, 376.22, E05.0) and autoimmune hypothyroidism (245, E06.3), noting that for physician claims only 3 digits were available. We generated lists of prescription medications available for the treatment of thyroid disease in Canada using the Anatomic Therapeutic Chemical system [17], including H03AA01, H03AA05, H03BA02, H03BA03, H03BB02 and H03CA. We did not include H03AA02 (liothyronine) since this medication is also used to treat depression. To improve the specificity of our case definition for AIT, we excluded persons with any ICD-9/10 diagnostic code for a thyroid neoplasm $(193$, C73, D34, D440) or with a physician tariff code or Canadian Classification for Health Intervention codes indicating thyroid surgery $(4911,4912,4917,4914,4925$, 4924, 4941, 1.FU.52., 1.FU.87., 1.FU.89. and 1.FU.91.). We also excluded persons taking lithium (Anatomic Therapeutic Chemical code N05AN01) because this can cause hypothyroidism. Finally, 
we developed several administrative case definitions, varying the number of physician, hospital and prescription claims required and the number of years used to classify a person as having AIT.

In the validation cohort, we compared the classification of AIT cases according to the administrative case definitions to medical records review by estimating the sensitivity, specificity, positive predictive value and negative predictive value. Youden's J, an index that equally weights sensitivity and specificity, was also calculated as follows: (sensitivity + specificity) - 1 [18]. Because there is no established gold standard for comorbidity measurement, we also calculated a $\kappa$ statistic for the agreement between administrative and medical records data. We interpreted agreement using $\kappa$ as follows: slight $(0-0.20)$, fair $(0.21-0.40)$, moderate $(0.41-$ $0.60)$, substantial (0.61-0.80) and almost perfect (0.81-1.0) [19].

\section{Prevalence}

We applied selected case definitions to the MS and general population cohorts. Once a person met the case definition, he or she was defined as affected in all subsequent years while alive and resident in Manitoba [20]. We determined the point prevalence on 1 October each year using the mid-year population figures from the $\mathrm{MH}$ population registry for denominators in the calculations. The population registry is maintained by $\mathrm{MH}$ and is updated when an individual moves into or out of Manitoba or dies. To estimate incidence, we required a 5 -year run-in period preceding the first AIT claim to ensure that cases were truly incident. In the MS cohort, an AIT case was defined as incident only if the first AIT claim occurred after the date of MS diagnosis, while in the matched cohort, an AIT case was defined as incident only if the first AIT claim occurred after the index date assigned based on their matched case. We used the direct method to age-standardize the results to the 2001 Canadian population [21] and calculated 95\% confidence intervals (CIs) assuming a Poisson distribution. Using Poisson regression, we calculated prevalence ratios (PRs), incidence rate ratios and 95\% CIs between the MS and control groups, adjusting for sex, age and year. Cell sizes $\leq 5$ were suppressed.

\section{Approvals}

Ethics approval was obtained from the University of Manitoba Health Research Ethics Board, and approval for administrative data access was obtained from the Manitoba Health Information Privacy Committee. To protect confidentiality, data linkage was performed via scrambled PHIN, using anonymized versions of the administrative databases. Written informed consent was obtained from all participants in the validation cohort. Statistical analyses used SAS 9.2 (SAS Institute Inc., Cary, N.C., USA).

\section{Results}

\section{Participants}

The study population included 4,192 persons with MS (71.7\% female) and 20,940 persons from the general population ( $71.7 \%$ female). Of the 430 persons with MS in the validation cohort, most were white $(391,91.6 \%)$ and women $(331,77.0 \%)$. They had a mean age at symptom onset of 33.2 years (SD 11.1). Based on medical records review, the frequency of AIT was 7.3\% ( $\mathrm{n}=30)$, and based on selfreport, it was 5.4\% $(n=23)$. Agreement between self-report and medical records for AIT was 'substantial', with an estimated $\kappa$ of 0.71 (95\% CI $0.57-0.86)$.

\section{Case Definitions}

We tested several administrative case definitions (labeled A to T) for AIT (table 1). The most striking finding was the substantial impact on sensitivity of adding prescriptions to the case definitions. For example, definition A used $\geq 1$ hospital or $\geq 2$ physician claims in 1 year to define AIT and had a sensitivity of only $5.9 \%$ with $100 \%$ specificity. Changing this definition to also include $\geq 2$ prescription claims or $\geq 1$ hospital or $\geq 2$ physician claims (definition $\mathrm{C}$ ) increased the sensitivity more than 10 -fold to $67.6 \%$, with a negligible reduction in specificity to $99.2 \%$. This is also illustrated in figure 1 , where the prevalence of AIT rises markedly in 1996 when prescription claims were first introduced, and in figure 2, where the incidence of AIT increases dramatically in 1996-1997 before stabilizing.

Agreement between case definition A and medical records was slight $(\kappa=0.10)$, but it was substantial between case definition $\mathrm{C}$ and medical records $(\kappa=0.75)$. Changing definition $\mathrm{A}$ to include 5 years of data (i.e. definition Q) improved sensitivity, but this still remained quite low at $17.6 \%$. The case definitions with the highest values of $\kappa(0.75)$ and Youden's J (0.72) were $\mathrm{O}$ and $\mathrm{S}$, requiring $\geq 1$ hospital or $\geq 2$ physician or $\geq 2$ prescription claims over 4 and 5 years, respectively.

\section{Prevalence}

To estimate the prevalence of AIT in the MS and general populations, we applied definition $S$, focusing on the period from 1996 onward when prescription claims were available. In 2005, the age-adjusted prevalence of thyroid disease was $9.51 \%$ (95\% CI 8.46-10.6) in the MS population and $8.58 \%$ (95\% CI 8.12-9.03) in the general population. The prevalence of thyroid disease increased with age and was higher in women than men (table 2).

As the results of Poisson modeling suggested an interaction between age group and population, we stratified the analysis. Among persons aged 20-44 years, the prevalence of AIT was slightly higher in the MS population than the general population (PR 1.25, 95\% CI 1.15-1.37). Similarly, among persons aged 45-59 years, the prevalence of thyroid disease was slightly higher in the MS than the general population (PR 1.25, 95\% CI 1.17-1.33). Among persons aged 60 years and older, the prevalence of AIT was slightly lower in the MS population (PR 0.88, 
Table 1. AIT: sensitivity, specificity, positive predictive value, negative predictive value and $\kappa$ of administrative claim case definitions compared to medical records review

\begin{tabular}{|c|c|c|c|c|c|c|c|c|}
\hline \multirow[t]{2}{*}{ Name } & \multicolumn{2}{|c|}{ Case definition } & \multirow[t]{2}{*}{ Sensitivity, \% } & \multirow[t]{2}{*}{ Specificity, \% } & \multirow[t]{2}{*}{ PPV, \% } & \multirow[t]{2}{*}{ NPV, \% } & \multirow[t]{2}{*}{$\kappa$} & \multirow[t]{2}{*}{ Youden's J } \\
\hline & $\begin{array}{l}\text { number of } \\
\text { years } \\
\text { of data }\end{array}$ & $\begin{array}{l}\text { number and type } \\
\text { of claims }\end{array}$ & & & & & & \\
\hline A & 1 & $\geq 1 \mathrm{H}$ or $\geq 2 \mathrm{P}$ & $5.9(0.72,19.7)$ & $100(99.0,100)$ & $100(15.8,100)$ & $92.1(89.0,94.5)$ & $0.10(-0.029,0.23)$ & 0.059 \\
\hline B & 1 & $\geq 1 \mathrm{H}$ or $\geq 1 \mathrm{P}$ & $5.9(0.72,19.7)$ & $100(99.0,100)$ & $100(15.8,100)$ & $92.1(89.0,94.5)$ & $0.10(-0.029,0.23)$ & 0.059 \\
\hline $\mathrm{C}$ & 1 & $\geq 1 \mathrm{H}$ or $\geq 2 \mathrm{P}$ or $\geq 2 \mathrm{Rx}$ & $67.6(49.5,82.6)$ & $99.2(97.7,99.8)$ & $88.5(69.8,97.6)$ & $97.1(94.9,98.5)$ & $0.75(0.62,0.87)$ & 0.67 \\
\hline $\mathrm{D}$ & 1 & $\geq 1 \mathrm{H}$ or $\geq 1 \mathrm{P}$ or $\geq 1 \mathrm{Rx}$ & $70.6(52.5,84.9)$ & $99.2(97.7,99.8)$ & $88.9(70.8,97.6)$ & $97.4(95.2,98.7)$ & $0.77(0.65,0.89)$ & 0.70 \\
\hline $\mathrm{E}$ & 2 & $\geq 1 \mathrm{H}$ or $\geq 2 \mathrm{P}$ & $11.8(3.30,27.4)$ & $99.5(98.1,99.9)$ & $66.7(22.3,95.7)$ & $92.5(89.4,94.9)$ & $0.18(0.019,0.34)$ & 0.11 \\
\hline $\mathrm{F}$ & 2 & $\geq 1 \mathrm{H}$ or $\geq 1 \mathrm{P}$ & $14.7(4.95,31.0)$ & $99.2(97.7,99.8)$ & $62.5(24.5,91.5)$ & $92.7(89.7,95.1)$ & $0.21(0.046,0.38)$ & 0.14 \\
\hline G & 2 & $\geq 1 \mathrm{H}$ or $\geq 2 \mathrm{P}$ or $\geq 2 \mathrm{Rx}$ & $70.6(52.5,84.9)$ & $98.4(96.5,99.4)$ & $80.0(61.4,92.3)$ & $97.3(95.1,98.7)$ & $0.73(0.60,0.86)$ & 0.69 \\
\hline $\mathrm{H}$ & 2 & $\geq 1 \mathrm{H}$ or $\geq 1 \mathrm{P}$ or $\geq 1 \mathrm{Rx}$ & $70.6(52.5,84.9)$ & $97.6(95.4,98.9)$ & $72.7(54.5,86.7)$ & $97.3(95.1,98.7)$ & $0.69(0.56,0.82)$ & 0.68 \\
\hline I & 3 & $\geq 1 \mathrm{H}$ or $\geq 2 \mathrm{P}$ & $11.8(3.30,27.4)$ & $99.5(98.1,99.9)$ & $66.7(22.3,95.7)$ & $92.5(89.4,94.9)$ & $0.18(0.019,0.34)$ & 0.11 \\
\hline J & 3 & $\geq 1 \mathrm{H}$ or $\geq 1 \mathrm{P}$ & $14.7(4.95,31.1)$ & $99.2(97.7,99.8)$ & $62.5(24.5,91.5)$ & $92.7(89.7,95.0)$ & $0.21(0.046,0.38)$ & 0.14 \\
\hline K & 3 & $\geq 1 \mathrm{H}$ or $\geq 2 \mathrm{P}$ or $\geq 2 \mathrm{Rx}$ & $70.6(52.5,84.9)$ & $98.4(96.5,99.4)$ & $80.0(61.4,92.3)$ & $97.3(95.1,98.7)$ & $0.73(0.60,0.86)$ & 0.69 \\
\hline $\mathrm{L}$ & 3 & $\geq 1 \mathrm{H}$ or $\geq 1 \mathrm{P}$ or $\geq 1 \mathrm{Rx}$ & $70.6(52.5,84.9)$ & $97.6(95.4,98.9)$ & $72.7(54.5,86.7)$ & $97.3(95.1,98.7)$ & $0.69(0.56,0.82)$ & 0.68 \\
\hline M & 4 & $\geq 1 \mathrm{H}$ or $\geq 2 \mathrm{P}$ & $17.6(6.76,34.5)$ & $99.5(98.1,99.9)$ & $75.0(34.9,96.8)$ & $92.9(90.0,95.3)$ & $0.26(0.087,0.44)$ & 0.17 \\
\hline $\mathrm{N}$ & 4 & $\geq 1 \mathrm{H}$ or $\geq 1 \mathrm{P}$ & $20.6(8.70,37.9)$ & $98.9(97.3,99.7)$ & $63.6(30.8,89.1)$ & $93.1(90.2,95.4)$ & $0.28(0.11,0.46)$ & 0.19 \\
\hline $\mathrm{O}$ & 4 & $\geq 1 \mathrm{H}$ or $\geq 2 \mathrm{P}$ or $\geq 2 \mathrm{Rx}$ & $73.5(55.6,87.1)$ & $98.4(96.5,99.4)$ & $80.6(62.5,92.5)$ & $97.6(95.5,98.9)$ & $0.75(0.63,0.87)$ & 0.72 \\
\hline$P$ & 4 & $\geq 1 \mathrm{H}$ or $\geq 1 \mathrm{P}$ or $\geq 1 \mathrm{Rx}$ & $73.5(55.6,87.1)$ & $97.6(95.4,98.9)$ & $73.5(55.6,87.1)$ & $97.6(95.4,98.9)$ & $0.71(0.58,0.84)$ & 0.71 \\
\hline Q & 5 & $\geq 1 \mathrm{H}$ or $\geq 2 \mathrm{P}$ & $17.6(6.76,34.5)$ & $99.5(98.1,99.9)$ & $75.0(34.9,96.8)$ & $92.9(90.0,95.3)$ & $0.26(0.087,0.44)$ & 0.17 \\
\hline $\mathrm{R}$ & 5 & $\geq 1 \mathrm{H}$ or $\geq 1 \mathrm{P}$ & $20.6(8.70,37.9)$ & $98.4(96.5,99.4)$ & $53.8(25.1,80.8)$ & $93.1(90.1,95.4)$ & $0.26(0.092,0.44)$ & 0.19 \\
\hline S & 5 & $\geq 1 \mathrm{H}$ or $\geq 2 \mathrm{P}$ or $\geq 2 \mathrm{Rx}$ & $73.5(55.6,87.1)$ & $98.4(96.5,99.4)$ & $80.6(62.5,92.5)$ & $97.6(95.5,98.9)$ & $0.75(0.63,0.87)$ & 0.72 \\
\hline $\mathrm{T}$ & 5 & $\geq 1 \mathrm{H}$ or $\geq 1 \mathrm{P}$ or $\geq 1 \mathrm{Rx}$ & $73.5(55.6,87.1)$ & $96.8(94.4,98.3)$ & $67.6(50.2,82.0)$ & $97.6(95.4,98.9)$ & $0.67(0.55,0.80)$ & 0.70 \\
\hline
\end{tabular}

Values in parentheses represent 95\% CIs. Prescription claim data were available from 1996 onward. PPV = Positive predictive value; NPV = negative predictive value; $\mathrm{H}$ = hospital; $\mathrm{P}=$ physician; $\mathrm{Rx}=$ prescription.

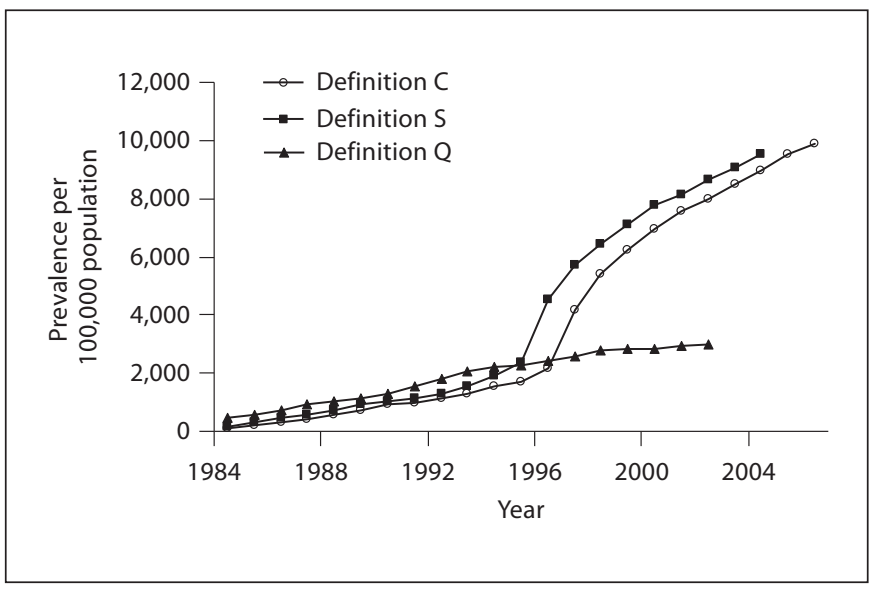

Fig. 1. Age-standardized prevalence of AIT in Manitobans with MS per 100,000 population. Definition C: $\geq 1$ hospital or $\geq 2$ physician or $\geq 2$ prescription claims in 1 year. Definition Q: $\geq 1$ hospital or $\geq 2$ physician claims in 5 years. Definition $S$ : $\geq 1$ hospital or $\geq 2$ physician or $\geq 2$ prescription claims in 5 years.

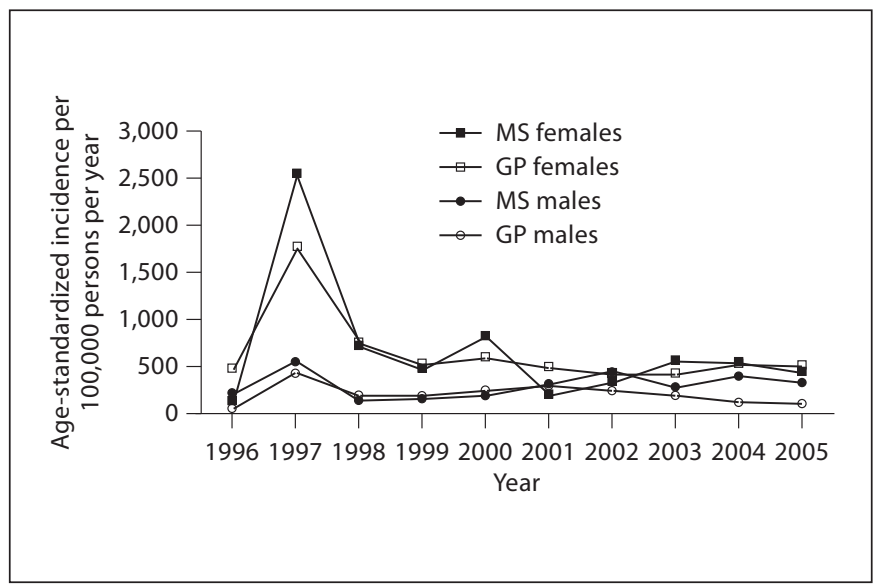

Fig. 2. Incidence of thyroid disease in the MS population and general population (GP), according to sex and year, per 100,000 persons per year. 
Table 2. Prevalence with $95 \%$ CIs of AIT in Manitoba per 100 population in 2006 according to age and sex

\begin{tabular}{|c|c|c|c|c|c|c|c|c|c|c|c|c|}
\hline Age & \multicolumn{3}{|c|}{ females } & \multicolumn{3}{|c|}{ males } & \multicolumn{3}{|c|}{ females } & \multicolumn{3}{|c|}{ males } \\
\hline $20-44$ years & 73 & 8.98 & $7.14,11.3$ & - & 2.22 & $0.92,5.34$ & 299 & 7.62 & $6.80,8.53$ & 11 & 1.03 & $0.57,1.86$ \\
\hline $45-59$ years & 152 & 13.6 & $11.6,15.9$ & - & 5.54 & $3.65,8.42$ & 620 & 11.4 & $10.6,12.4$ & 49 & 2.58 & $1.95,3.42$ \\
\hline
\end{tabular}

*Age groups were collapsed because cell sizes $\leq 5$ were suppressed. prev = Prevalence; - = suppressed.

95\% CI 0.85-0.92). During the interval 1996-2005, the age- and sex-adjusted prevalence of AIT rose similarly in both populations each year, with a PR per year of 2.91 (95\% CI 2.29-3.70) in the MS population and 2.60 (95\% CI 2.09-3.24) in the general population.

\section{Incidence}

Given that definition S required prescription claims which were not available until 1996, and that we wanted a run-in period to ensure that we were truly capturing incident cases, we estimated the incidence of AIT for the years 2001-2005. In 2005, the age-adjusted incidence of AIT per 100,000 persons per year was 422.9 (95\% CI 204.4-641.4) in the MS population and 397.8 (95\% CI 299.0-496.5) in the general population. Over the interval 2001-2005, the incidence of thyroid disease did not differ between the MS and general populations after adjusting for age, sex and year (incidence rate ratio $0.98,95 \%$ CI 0.73-1.30; fig. 2).

Among incident cases, the mean age of onset of AIT in the MS population was 54.8 years (SD 13.1), more than 3 years earlier than in the general population [ 57.8 years $(\mathrm{SD} 14.0) ; \mathrm{p}=0.018]$.

\section{Discussion}

Administrative data are gathered during the routine delivery of health care and have been used by researchers for multiple purposes $[11,22,23]$. They may be particularly useful for evaluating the impact of rare comorbidities on a population and when the outcomes of interest relate to mortality and health care utilization, for which substantial experience with such data already exists. However, the accuracy of case definitions for chronic dis- ease varies widely according to the condition [17, 24], making it important to validate case definitions prior to use.

Our first study aim was to validate an administrative case definition of AIT. All definitions tested produced high specificity, exceeding $97 \%$. However, to achieve definitions with adequate sensitivity and substantial agreement with medical records, we had to incorporate the use of prescription claims. This likely reflects the fact that once the diagnosis of AIT is established and treatment is initiated, few health care encounters will occur where AIT is listed as the primary reason for the encounter. This raises the possibility that prescription claims may not be needed to identify incident cases, but additional work in a larger population would be needed to evaluate this possibility. Our proposed case definition appears to be adequate for surveillance of AIT in jurisdictions with prescription claims; however, without prescription claims the prevalence of AIT would be substantially underestimated.

The best method for identifying comorbidity in persons with MS has not been established, and this may vary by comorbidity [25]. At $10.4 \%$, the crude prevalence of AIT was highest using administrative data, followed by 7.3\% for medical records and lowest for self-report at $5.4 \%$. Agreement between the three data sources was substantial, being $\kappa=0.71$ for agreement between self-report and medical records, $\kappa=0.75$ for agreement between administrative data and medical records, and $\kappa=0.67$ for agreement between administrative data and self-report. The frequency of AIT according to medical records may be lower than that obtained from administrative data because we reviewed medical records available at our tertiary care centre and in local neurologists' offices but did not review all medical records of all providers. 
The second study aim was to estimate the prevalence and incidence of AIT in persons with MS and in the general population. Our findings for the general population were similar to those reported in the Colorado Thyroid Prevalence Study [26], as well as in a study performed using prescription claims data in the province of Quebec, Canada. The latter study reported a prevalence of thyroid hormone use in women of $10.8 \%$ and in men of $2.9 \%$ in 2001 [27]. The prevalence of AIT in the Manitoba MS population was $9.5 \%$. Prior studies reported that the prevalence of thyroid disease in MS ranged from 0.92 to $13.9 \%$, but population-based studies in North America and Europe found that the prevalence was 7.9-8.6\%, similar to our findings. A population-based study in Taiwan, however, reported somewhat different findings. That study focused on hypothyroidism alone, rather than hypo- and hyperthyroidism, and found that only $1.7 \%$ of persons with MS were affected. This may reflect two factors. Firstly, the study population was ethnic Chinese with a potentially lower underlying risk of AIT, given that only $0.5 \%$ of the general population controls had hypothyroidism. Secondly, the investigators used only 2 years of data and did not incorporate prescription claims into their case definition, potentially reducing the sensitivity of their case definition as compared to the case definition used in the present study.

Little information is available regarding the incidence of AIT in MS, and most of this information is drawn from clinical trials or observational studies focused on the influence of interferon-beta on AIT. In contrast to prior studies, we were able to examine the incidence of AIT in an unselected MS population. The incidence of AIT was 422.8 per 100,000 persons per year and was stable over time. Based on the date of the first claim for AIT, we found an earlier age of onset in the MS population than the general population. It is uncertain whether this reflects a true difference in the age of onset between the two populations or differences in ascertainment. Routine testing of thyroid function is recommended for persons with MS taking interferon-beta, and guidelines for managing fatigue in MS encourage the exclusion of secondary causes of fatigue such as thyroid dysfunction [28]. Thus, persons with MS may undergo more testing for thyroid disease, leading to earlier diagnosis.

Overall, the incidence and prevalence of AIT did not differ between the MS and general populations. Prior studies were inconsistent with respect to a possible increased risk of AIT in the MS population [2, 4, 29]. Among studies which were population-based, one did not find an increased risk, one with small sample sizes found an in- creased risk of Graves' disease but not Hashimoto's disease, while the Taiwanese study found a threefold increased risk of hypothyroidism in MS [4-6]. However, the latter study had a much lower prevalence of AIT than reported here. In both populations, the incidence of AIT was stable over time, but the prevalence rose. Studies in the general population have noted similar findings, but we were unable to find comparable studies for the MS population [27].

In addition to not reviewing the medical records of all providers, this study had other limitations. We did not distinguish between hypo- and hyperthyroidism due to small numbers of affected persons with MS. Small numbers also limited us to examining incidence and prevalence using only three age groups, so that we were unable to examine variations in disease risk and burden in detail across age groups, including whether AIT prevalence continues to rise after the age of 60 years. Finally, we did not evaluate the incidence or prevalence of AIT in relation to the use of disease-modifying therapy as this was beyond the scope of the approvals for the use of administrative data and requires a different study design and analytical technique. The strengths of this study included validation of the administrative case definition in a cohort with similar characteristics to the population in which it was ultimately applied, and the populationbased design.

We developed and tested an administrative case definition of AIT and showed that it performs adequately for disease surveillance and pharmacovigilance studies. In earlier work, we showed that administrative data can be used for surveillance of diabetes, hypertension and hyperlipidemia, suggesting that administrative data have utility for surveillance of a range of comorbidities in MS [30]. Development and validation of case definitions for other conditions, including those which are common in the MS population and those which may occur as adverse effects of therapy, should be pursued as a cost-effective approach to pharmacovigilance. This will be of increasing importance as novel therapies which may increase the risk of AIT and other comorbidities become available [9]. The incidence and prevalence of AIT were similar in the MS and general populations. Future studies will need to verify the applicability of the proposed case definition for MS in other jurisdictions and in other chronic disease populations. It will also be important to specifically evaluate the incidence of AIT in persons treated with approved and emerging therapies for MS to enhance our knowledge of their risk-benefit profiles. 


\section{Acknowledgements}

This study was supported (in part) by grants from the Multiple Sclerosis Society of Canada and the Manitoba Health Research Council.

\section{Disclosure Statement}

Ruth Ann Marrie receives research funding from the Canadian Institutes of Health Research, Public Health Agency of Canada, Manitoba Health Research Council, Health Sciences Centre Foundation, Multiple Sclerosis Society of Canada, Multiple Sclerosis Scientific Foundation and Rx \& D Health Research Foundation and has conducted clinical trials funded by Bayer Inc. and Sanofi-Aventis.

Nancy Yu receives research support from the Canadian International Development Agency, the Multiple Sclerosis Society of Canada, Canadian Institutes of Health Research and Manitoba Health and Healthy Living.

Stella Leung reports no disclosures.

Lawrence Elliott receives research support from the Canadian Institutes of Health Research, Health Sciences Centre Foundation, Public Health Agency of Canada and Multiple Sclerosis Society of Canada.

Sharon Warren receives research funding from the Canadian Institutes of Health Research, Canadian Health Services Research Foundation, Alberta Health Services and Social Sciences and Humanities Research Council of Canada.
Christina Wolfson receives research funding from the Multiple Sclerosis Society of Canada, Canadian Institutes of Health Research, Canada Foundation for Innovation and Public Health Agency of Canada.

Helen Tremlett currently receives funding from the Multiple Sclerosis Society of Canada (Don Paty Career Development Award), US National MS Society [RG 4202-A-2 (PI)], Canadian Institutes of Health Research [MOP 190898 (PI) and MOP-93646 (PI)], Michael Smith Foundation for Health Research (Scholar award) and the Canada Research Chair program. She has received speaker's honoraria and/or travel expenses to attend conferences from the Consortium of MS Centres, US National MS Society, Swiss Multiple Sclerosis Society, University of British Columbia Multiple Sclerosis Research Program, Teva Pharmaceuticals and Bayer Pharmaceuticals (honoraria declined) and the European Committee for Treatment and Research in Multiple Sclerosis. Unless otherwise stated, all speaker honoraria are either donated to an MS charity or to an unrestricted grant for use by her research group.

John Fisk is the Director of the endMS Atlantic Regional Research and Training Centre which is funded by the Multiple Sclerosis Society of Canada. He receives research funding from the Canadian Institutes of Health Research and in the past has received grants, honoraria and consultation fees from AstraZeneca, Bayer, Biogen Idec Canada, Heron Evidence Development Limited, Hoffmann-La Roche, MAPI Research Trust, Novartis, Sanofi-Aventis, Serono Canada and QualityMetric Incorporated.

James Blanchard receives research support from the Multiple Sclerosis Society of Canada, Canadian Institutes of Health Research, Bill \& Melinda Gates Foundation, Canadian International Development Agency and United States Agency for International Development.

\section{References}

1 Baker HWG, Balla JI, Burger HG, Ebeling P, Mackay IR: Multiple sclerosis and autoimmune diseases. Aust NZ J Med 1972;2:256260.

-2 Seyfert S, Klapps P, Meisel C, Fischer T, Junghan U: Multiple sclerosis and other immunologic diseases. Acta Neurol Scand 1990;81: $37-42$.

- 3 Edwards LJ, Constantinescu CS: A prospective study of conditions associated with multiple sclerosis in a cohort of 658 consecutive outpatients attending a multiple sclerosis clinic. Mult Scler 2004;10:575-581.

4 Kang J-H, Chen Y-H, Lin H-C: Comorbidities amongst patients with multiple sclerosis: a population-based controlled study. Eur J Neurol 2010;17:1215-1219.

-5 Sloka JS, Pryse-Phillips WEM, Stefanelli M, Joyce C: Co-occurrence of autoimmune thyroid disease in a multiple sclerosis cohort. J Autoimmune Dis 2005;2:9.

-6 Wynn DR, Rodriguez M, O'Fallon WM, Kurland LT: A reappraisal of the epidemiology of multiple sclerosis in Olmsted County, Minnesota. Neurology 1990;40:780-786.
-7 Caraccio N, Dardano A, Manfredonia F, Manca L, Pasquali L, Iudice A et al: Longterm follow-up of 106 multiple sclerosis patients undergoing Interferon- $\{$ beta $\} 1 \mathrm{a}$ or $1 \mathrm{~b}$ therapy: predictive factors of thyroid disease development and duration. J Clin Endocrinol Metab 2005;90:4133-4137.

-8 CAMMS223 Trial Investigators, Coles AJ, Compston DA, Selmaj KW, Lake SL, Moran S, Margolin DH, Norris K, Tandon PK: Alemtuzumab vs. interferon beta-1a in early multiple sclerosis. N Engl J Med 2008;359: 1786-1801.

-9 Cossburn M, Pace AA, Jones J, Ali R, Ingram G, Baker K, et al: Autoimmune disease after alemtuzumab treatment for multiple sclerosis in a multicenter cohort. Neurology 2011; 77:573-579.

10 Hazell L, Shakir SAW: Under-reporting of adverse drug reactions: a systematic review. Drug Saf 2006;29:385.

1 Tricco AC, Pham B, Rawson NSB: Manitoba and Saskatchewan administrative health care utilization databases are used differently to answer epidemiologic research questions. J Clin Epidemiol 2008;61:192-197.e112.
12 Health Information Management Branch: Population Report. Winnipeg, Manitoba Health and Healthy Living, 2008.

13 Marrie RA, Yu N, Blanchard JF, Leung S, Elliott $\mathrm{L}$ : The rising prevalence and changing age distribution of multiple sclerosis in Manitoba. Neurology 2010;74:465-471.

14 Horton M, Rudick RA, Hara-Cleaver C, Marrie RA: Validation of a self-report comorbidity questionnaire for multiple sclerosis. Neuroepidemiology 2010;35:83-90.

- 15 Polman CH, Reingold SC, Edan G, Filippi M, Hartung HP, Kappos L, et al: Diagnostic criteria for multiple sclerosis: 2005 revisions to the 'McDonald Criteria'. Ann Neurol 2005; 58:840-846.

16 Canadian Cancer Society's Steering Committee on Cancer Statistics: Canadian Cancer Statistics 2011. Toronto, Canadian Cancer Society, 2011.

17 Lix L, Yogendran M, Shaw S, Burchill C, Metge C, Bond R: Population-based data sources for chronic disease surveillance. Chronic Dis Can 2008;29:22-30.

18 Youden WJ: Index for rating diagnostic tests. Cancer 1950;3:32-35. 
19 Landis JR, Koch GG: The measurement of observer agreement for categorical data. Biometrics 1977;33:159-174.

20 National Diabetes Surveillance System: Responding to the Challenge of Diabetes in Canada. Ottawa, Health Canada, 2003.

21 Rothman KJ, Greenland S (eds): Modern Epidemiology, ed 2. Philadelphia, Lippincott Williams \& Wilkins, 1998.

22 Berlowitz DR, Hoenig H, Cowper DC, Duncan PW, Vogel WB: Impact of comorbidities on stroke rehabilitation outcomes: does the method matter? Arch Phys Med Rehabil 2008;89:1903-1906.

-23 Longobardi T, Bernstein CN: Utilization of health-care resources by patients with IBD in Manitoba: a profile of time since diagnosis. Am J Gastroenterol 2007;102:1683-1691.
24 Lix L, Yogendran M, Shaw S, Targownick L, Jones J, Bataineh O: Comparing administrative and survey data for ascertaining cases of irritable bowel syndrome: a populationbased investigation. BMC Health Serv Res 2010;10:31.

25 Marrie RA, Horwitz RI: Emerging effects of comorbidities on multiple sclerosis. Lancet Neurol 2010;9:820-828.

26 Canaris GJ, Manowitz NR, Mayor G, Ridgway EC: The Colorado Thyroid Disease Prevalence Study. Arch Intern Med 2000; 160:526-534.

27 Gagnon F, Langlois M-F, Michaud I, Gingras S, Duchesne J-F, Lévesque B: Spatio-temporal distribution of hypothyroidism in Quebec. Chronic Dis Can 2006;27:1-8.
28 Multiple Sclerosis Council for Clinical Practice Guidelines: Fatigue and Multiple Sclerosis: Evidence-Based Management Strategies for Fatigue in Multiple Sclerosis. Washington, Paralyzed Veterans of America, 1998.

29 Ramagopalan SV, Dyment DA, Valdar W, Herrera BM, Criscuoli M, Yee IM, et al: The occurrence of autoimmune disease in Canadian families with multiple sclerosis. Lancet Neurol 2007;6:604-610.

30 Marrie RA, Yu BN, Leung S, Elliott L, Caetano P, Warren S, Wolfson C, Patten SB, Svenson LW, Tremlett H, Fisk J, Blanchard JF: Rising prevalence of vascular comorbidities in MS: validation of administrative definitions for diabetes, hypertension, and hyperlipidemia. Mult Scler 2012; Feb 10., E-pub ahead of print. 